\title{
Les controverses politiques et leurs frontières
}

Political Controversies and Their Boundaries

\section{Juliette Rennes}

\section{(2) OpenEdition}

\section{Journals}

Édition électronique

URL : http://journals.openedition.org/edc/6614

DOI : $10.4000 /$ edc. 6614

ISSN : 2101-0366

Éditeur

Université Lille-3

\section{Édition imprimée}

Date de publication : 1 décembre 2016

Pagination : $21-48$

ISBN : 978-2-917562-16-1

ISSN : $1270-6841$

Référence électronique

Juliette Rennes, "Les controverses politiques et leurs frontières », Études de communication [En ligne], 47 | 2016, mis en ligne le 01 décembre 2018, consulté le 01 mai 2019. URL : http://

journals.openedition.org/edc/6614; DOI : 10.4000/edc.6614 


\section{Les controverses politiques}

et leurs frontières

Political Controversies and Their Boundaries

Juliette Rennes

École des hautes études en sciences sociales, CEMS - IMM/UMR-8178 Centre d'étude des mouvements sociaux - Institut Marcel Mauss rennes@ehess.fr 
Résumé / Abstract

À partir d'une discussion des travaux sur les échanges agonistiques et de recherches empiriques réalisées sur les controverses, cet article explore la distinction entre les controverses et des formes parentes, notamment le débat et la polémique, puis précise en quoi consistent les frontières et les recouvrements entre controverses scientifiques et politiques, et enfin entre les controverses politiques et ces autres formes de conflictualité politique que sont « l'affaire » et le « problème public ». Ce travail de clarification ne vise pas à substantialiser ces distinctions, mais à rendre comparables différents débats empiriques, à mieux saisir en quoi consiste le lien entre conflictualité et politisation et, enfin, à explorer les limites de l'approche de la conflictualité politique à partir de l'analyse des formes publicisées de débats.

Mots-clés : controverse, polémique, débat, affaire, problème public, corpus, hégémonie.
In this article, I explore how certain controversies become political. Firstly, I analyse the boundaries and overlaps of three forms of agonistic exchange: debate, polemic and controversy. I then distinguish different kinds of controversies according to their empirical dimension and according to the status of the actors. Finally, I distinguish political controversies from two other forms of political conflict: "case making" (affaire) and "public problem". Such clarifications do not seek to reify distinctions. The intention of this study is rather to render different empirical debates comparable and to better understand the link between conflict and politicisation.

Keywords: controversy, polemic, debate, public problem, hegemony, discourse analysis. 
Est-il juste, nécessaire, utile de dépénaliser l'avortement et la consommation de cannabis, de taxer les transactions financières, de contrôler la vente des armes à feu, d'accorder le mariage aux couples de même sexe et le droit de vote aux résidents étrangers, d'imposer des quotas de femmes dans les assemblées élues, d'abolir la peine de mort ? Pour désigner la dimension clivante de ces questions dans plusieurs pays au tournant des XX-XX|e siècles, les termes de " controverse ", " polémique ", " affaire ", " débat » ou éventuellement « problème » peuvent être successivement mobilisés dans les médias et les échanges ordinaires. Dans certains domaines des sciences humaines et sociales, ces termes désignent des phénomènes distincts sans que cette distinction ne soit pour autant stabilisée : une " polémique », par exemple, peut être définie et appréhendée sous des angles très différents en sociologie, en linguistique, en sciences de l'information et de la communication, en histoire ou au sein de divers courants de recherche internes à chacune de ces disciplines.

Dans les disciplines littéraires et linguistiques sont privilégiées l'étude des ressources rhétoriques, argumentatives et lexicales des discours agonistiques et l'analyse des genres discursifs dédiés à leur expression (pamphlet, satire, libelle, brûlot, lettre ouverte, manifeste...) ; tandis que les sciences de l'information et de la communication ont enrichi la compréhension des dispositifs médiatiques qui organisent les confrontations de points de vue. Dans les science studies, la dynamique des controverses portant sur des enjeux cognitifs et épistémologiques au sein de communautés scientifiques fait l'objet d'une importante littérature empirique et théorique depuis les années 1980. Au point que les travaux des science studies ont largement servi de modèle, dans l'ensemble de la sociologie, pour décrire toutes sortes de débats litigieux, y compris les débats politiques évoqués en ouverture de cet article qui se distinguent nettement des controverses scientifiques par leurs enjeux empiriques, leurs acteurs et leurs publics.

Dans cet article, je souhaite contribuer à clarifier les recouvrements et les frontières entre différentes formes agonistiques et entre les approches élaborées pour les décrire et les comprendre. J'essaye ensuite plus spécifiquement de circonscrire les questions litigieuses dont la formulation et les enjeux peuvent être qualifiés de "politiques ", qualification sur laquelle je reviendrai. Qu'implique, du point de vue épistémologique et méthodologique, le fait de les appréhender comme formant une controverse plutôt qu'une polémique, un débat, un différend, une affaire, une mobilisation ou un problème public ? Et quelles sont les dimensions de la conflictualité politique laissées dans l'ombre quand on délimite un corpus pour analyser une controverse publique ? Ayant été directement confrontée dans mes recherches à ces deux questions relatives à la fois à la catégorisation des différentes formes agonistiques publiques et à la délimitation du matériau d'enquête des controverses politiques, j'expose les 
analyses auxquelles je suis parvenue, tout en m'appuyant sur une discussion des travaux existants ${ }^{1}$.

1.

Débat, polémique, controverse

Dans une contribution à un ouvrage sur la parole polémique paru en 2003, Christian Plantin constatait que " débat ", " polémique " et " controverse » étaient couramment employés dans la presse comme synonymes au sein d'un même article (Plantin, 2003). Ce constat vaut toujours plus d'une décennie après, même si ces trois termes sont, à l'époque comme aujourd'hui, inégalement usités : " débat » est bien plus courant que «polémique ", lui-même beaucoup plus courant que " controverse $»^{2}$. De fait, " débat » est un terme générique large ou pauvre - un « hypergenre » selon les termes de Dominique Maingueneau ${ }^{3}$ - qui désigne simplement une confrontation argumentée de points de vue, quels que soient ses enjeux, son objet, sa situation d'énonciation, sa temporalité et son degré de conflictualité : un débat peut renvoyer à un échange unique ou mainte fois réitéré, oral ou écrit, focalisé sur une question bien déterminée ou embrassant une diversité de questions non définies d'avance. En outre, la nature des questions donnant matière à débat n'est circonscrite à aucun domaine d'activité et les différences entre les points de vue des débatteurs peuvent aller de simples nuances à des oppositions vécues comme insurmontables. Pour cet ensemble de raisons, le degré d'argumentativité d'un débat est très variable : lorsque l'enjeu du débat est considéré par ses protagonistes comme mineur et qu'ils/elles n'expérimentent pas leurs différences de points de vue comme des antagonismes, l'échange a peu de chance d'être structuré par ce que Marc Angenot a appelé des « arsenaux argumentatifs » (Angenot, 2012), soit des argumentaires déjà éprouvés, stabilisés et consolidés par leur(s) confrontation(s) antérieure(s) avec une série d'arguments adverses sur le même objet.

Néanmoins, fut-elle réduite, c'est bien la présence d'arguments, et même d'un certain type d'arguments relevant d'une " grammaire " de la vie publique, qui distingue le débat d'autres formes dialogales non agonistiques comme la

1 Un grand merci à German Fernandez Vavrik et Sezin Topçu pour les discussions que nous avons eues sur les controverses et qui ont contribué à nourrir cet article.

2 Dans la base d'articles d'Europresse (1088 sources de presse en français), du 25 avril 2006 au 25 avril 2016, il y a 4,6 fois plus de titres d'articles avec le syntagme " débat sur » (41 547 articles) que « polémique sur » ( 8890 articles), et 20,8 fois plus que " controverse sur » (1995 articles). Cette moindre présence du terme " controverse » apparaît également sur un corpus thématique lié aux débats sur le climat (Scotto d'Apollonia et al., 2014).

3 Un hypergenre est un mode d'organisation textuelle aux contraintes pauvres (" dialogue », " lettre », etc.), qu'on retrouve à des époques et dans des lieux très divers (Maingueneau, 2009 [1996], 73). 
simple conversation. Dans une conversation ordinaire, il est admis qu'un locuteur ou une locutrice justifie une disposition subjective par sa seule relation vécue et singulière envers la question posée (j'aime telle musique parce que mes parents l'écoutaient quand j'étais enfant). En revanche, pour qu'il y ait " débat ", il ou elle doit fournir des raisons d'adhérer, de préférer ou d'agir qui soient détachées de sa situation singulière (j'aime telle musique qui présente telle ou telle qualité). Et c'est précisément cette forme de "désingularisation " (Boltanski, 1984) qui permet de rendre une position susceptible d'être discutée.

Ce travail argumentatif est moins définitoire des échanges désignés comme constituant une " polémique " : dans le corpus de presse étudié par Christian Plantin, les journalistes optent pour ce terme lorsqu'ils perçoivent, dans une confrontation discursive sur une question donnée, des « émotions violentes de l'ordre de la colère ou de l'indignation " (Plantin, 2003, 406). Dans les travaux d'histoire littéraire et d'analyse rhétorique, que la polémique soit considérée comme une sous-catégorie de l'hypergenre "débat " ou comme un genre distinct, elle est toujours caractérisée par la " virulence » de l'expression des oppositions entre les polémistes (professionnels) ou entre les " polémiqueurs" (ordinaires) sur une question donnée (Kerbrat-Orecchioni, 1980 ; Amossy, 2014). Définie alors comme un type d'échange peu susceptible de déboucher sur un accord, la polémique est repérable, du point de vue linguistique et rhétorique, par des marqueurs d'oppositions, voire des formes de violences verbales (injure, insulte, vitupération, sarcasme...) qui tendent à emphatiser les antagonismes plutôt que par la présence d'arguments visant à donner prise à la discussion ${ }^{4}$.

Ainsi, les pamphlets, analysés par Angenot (1982) comme typiques de la posture polémique, tendent à présenter la/les position(s) adverse(s) sous une forme déjà condamnée, à travers une rhétorique assertive et fortement modalisée. Certes, on peut identifier, dans ce type d'écrits, une dimension argumentative (Amossy, 2008, 4), au sens où les pamphlétaires, par l'expression verbale de leurs jugements et de leurs émotions, visent bel et bien à orienter axiologiquement la représentation que le lectorat se fait de l'énonciateur et de sa cible. Mais on ne saurait parler d'une structure argumentative : les pamphlétaires n'explicitent que marginalement les raisons du dissensus et ne s'efforcent pas de créer un espace commun d'échange agonistique avec leur(s) adversaire(s).

Si l'on s'intéresse à présent aux usages du terme " controverse " en sciences humaines et sociales, on retrouve certaines caractéristiques à la fois du débat et de la polémique, tout en pouvant distinguer au moins deux propriétés spécifiques à ce terme. La controverse, à l'instar de la polémique, désigne une confrontation discursive polarisée, c'est-à-dire à la fois focalisée sur un objet précis et suscitant des oppositions tranchées (Charaudeau, 2015, 15). Cette

4 Un « argument » est entendu ici comme un "énoncé (ou fragment de discours) vraisemblable qui exprime une raison avancée pour accréditer une proposition controversée, ayant le statut de conclusion " (Plantin, 2002, 65) 
double dimension, on l'a dit, n'est pas nécessairement définitoire d'un débat. La controverse apparaît donc comme une sous-catégorie de la forme débat dont elle partage les propriétés générales, tout en ayant comme propriété spécifique d'être polarisée. Mais elle se distingue également de la polémique par le fait que cette polarisation est, de part et d'autre, l'objet d'une argumentation poussée. Dans la plupart des travaux, décrire une controverse, qu'elle soit religieuse, esthétique, scientifique, littéraire, philosophique, sociotechnique ou politique implique de faire référence à un complexe d'arguments opposés.

Du point de vue des disciplines du discours, c'est précisément cette articulation d'une forte polarisation et d'une forte structure argumentative qui distingue la controverse d'autres échanges agonistiques : si la polarisation diminue entre les protagonistes, la controverse peut devenir une "discussion ", une " délibération », un "dialogue »; si, tout en demeurant vive, la polarisation cesse pourtant de s'appuyer sur la formulation explicite d'arguments pour basculer plutôt vers des procédés de disqualification de l'adversaire, on entre dans la « querelle », la « dispute », le « pugilat », ou justement, la « polémique ». Cette opposition des usages est mise en relation par certains chercheurs avec l'origine des termes : alors que " controversia " en latin est liée à la langue du tribunal renvoyant à une opposition régulée par l'appareil judiciaire (Desborde, 1995), " polemos », en grec, désigne la guerre : la connotation belliqueuse survit dans l'adjectif et le nom qui en sont issus. On retrouve cette distinction dans la caractérisation de la controverse comme débat "réglé » (Dascal, 1995, 2008 ; Amossy et Burger, 2011) se distinguant d'autres formes d'échanges polémiques qui consistent au contraire à transgresser - fut-ce de façon codifiée et prévisible - les normes formelles du raisonnement, du langage policé et du respect de l'adversaire. Ces différents registres discursifs peuvent bien sûr se succéder au sein d'un même débat concret et la dénomination d'un échange agonistique par ses protagonistes eux-mêmes peut osciller entre ces différents termes : reprocher à son adversaire de transformer la controverse en polémique est une stratégie ordinaire de disqualification de ses prises de positions ${ }^{5}$.

Confrontation discursive argumentée et polarisée, la controverse est aussi considérée, dans la plupart des travaux sur le sujet, comme une confrontation " prolongée ", " durable ", " réitérable ", " recyclable ", ou encore "suivie " (Benoit-Barné et McDonald, 2011 ; Dascal, op. cit., 1995, 2008 ; Eemeren et Garssen, 2008 ; Le Robert, 2001 ; Rennes, 2007a). Alors qu'un débat, une discussion, un désaccord, une dispute, une polémique, un litige, une délibération peuvent se limiter à une seule occurrence, parler d'une controverse, c'est faire référence à une série d'échanges agonistiques dont on considère qu'ils portent sur une même question.

5 Ces « jugements de polémicité » (Jacquin, 2011) ont été mis en relief dans de nombreux débats. Voir, par exemple, Chateauraynaud et Torny (1999) et Plantin (2003). 
Cette dimension suivie et réitérable a plusieurs implications pour la recherche sur une controverse. Premièrement, la temporalité elle-même de la controverse peut devenir un objet d'enquête : on s'intéresse alors à son émergence, sa publicisation, sa dispersion dans plusieurs arènes, ses phases de reflux, de renaissance, éventuellement de clôture (Lilti, 2007). Deuxièmement, les arguments d'un débat concret peuvent eux-mêmes être envisagés dans leur temporalité et leur " trajectoire » en relation avec les "épreuves» qu'ils traversent (Chateauraynaud, 2011) en étant successivement confrontés à des contre-arguments au cours d'autres débats concrets relevant de la même controverse. Troisièmement, au fil de la carrière d'une controverse, certains arguments, que l'on finit par considérer comme «typiques » d'une controverse, sont mainte fois mobilisés par une diversité d'acteurs sociaux dans des situations d'énonciation diverses tout à fait éloignées du contexte d'élaboration de ces arguments. Il convient alors, dans une enquête sur une controverse, de ne pas écraser ces différents niveaux de temporalité : celle des protagonistes participant et alimentant successivement la controverse et celle, souvent de plus longue durée, des formes argumentatives qu'ils se transmettent sans les avoir eux-mêmes élaborées (Rennes, 2011a).

Enfin, cette dimension suivie et réitérable de la controverse va de pair avec le caractère fortement métadiscursif et interdiscursif des échanges agnostiques qui la constituent : ses protagonistes tendent à inscrire ce qui les divise dans une histoire discursive, se référant, dans chaque débat, à d'autres débats antérieurs qu'ils présentent comme portant sur une même question litigieuse (I'avortement, le mariage des couples de même sexe, la peine de mort, les biotechnologies...). À l'inverse, un débat dont l'objet et l'enjeu apparaissent aux acteurs comme entièrement inédits est rarement catégorisé d'emblée comme relevant d'une controverse. En somme, dans une recherche, le fait que les protagonistes eux-mêmes d'un débat caractérisent celui-ci comme réactualisant une question clivante déjà débattue antérieurement peut faire partie des critères - nécessaires, mais non suffisants - permettant de qualifier leur échange comme s'inscrivant dans une " controverse ».

Outre son caractère polarisé, argumenté et réitérable, la controverse renvoie également, dans les sciences humaines et sociales, à des échanges agonistiques ayant une dimension " publique ». La publicité renvoie ici à une caractéristique à la fois morphologique (discursive) et situationnelle : sur le plan discursif, la « grammaire publique » désigne une contrainte argumentative, celle que nous avons mentionnée à propos d'un débat et qui consiste, pour les débatteurs, à énoncer des raisons d'agir, d'adhérer ou de rejeter qui, loin de se limiter à la seule expression de leur ressenti ou de leur intérêt singulier, doivent faire l'objet d'une opération de désingularisation. Sur le plan situationnel, la publicité fait référence à l'existence d'un auditoire potentiellement indéterminé : contrairement à un échange agonistique se déroulant exclusivement dans un espace interactionnel et perceptuel dont les membres contrôlent les frontières (par exemple un désaccord entre proches se déroulant dans la cuisine d'un 
appartement privé), on tend à qualifier de controverse un débat réitéré dans diverses situations de communication au sein desquelles les protagonistes n'ont pas toujours « de contrôle sur qui pourrait les observer et pas de moyen de sélectionner l'auditoire auquel ils s'adressent » selon la définition d'une « situation publique » que propose Joseph Gusfield (2009 [1981], 197).

Loin d'être une propriété figée, la publicité désigne ainsi un processus qui peut connaître différents degrés d'extension (Cardon, Heurtin, Lemieux, 1995) : une communication scientifique dans un colloque, une intervention militante dans un espace associatif, n'ont évidemment pas un auditoire aussi indéterminé, diversifié et étendu qu'une prise de parole parlementaire retransmise par tous les grands médias, mais elles n'en constituent pas moins, à des degrés divers, des situations de communication publique au sens où l'entend la sociologie des interactions.

Au terme de ce premier examen des usages du terme " controverse » en sciences humaines et sociales, on peut définir la controverse comme étant une confrontation discursive polarisée, argumentée, réitérée et publique. Cependant, ce socle définitionnel recouvre encore une grande diversité empirique de débats du point de vue de leur durée, leur périmètre, leur degré de publicité, leur situation d'énonciation, leurs protagonistes, leurs enjeux. Une confrontation argumentée et réitérée, portant sur l'interprétation d'un événement, d'un précepte religieux, d'une théorie, d'une doctrine, d'une œuvre d'art qui, sans être confinée à un espace " privé ", se déroule cependant au sein d'un groupe de " pairs » dans une institution scientifique, sportive, artistique, littéraire, ecclésiastique, et qui se tarit au bout de quelques mois, peut tout à fait entrer dans la définition énoncée ci-dessus de " controverse ". Mais c'est aussi le cas d'un débat de plusieurs décennies suscité par une revendication transformant fondamentalement une norme stato-nationale relative à la filiation, à la fiscalité ou à la liberté de circulation, qui se déploie dans une multiplicité d'arènes (Parlement, gouvernement, presse, université, associations...) et qui, au fil de sa carrière, implique différentes générations de débatteurs, lesquels " relancent » successivement une question clivante qu'ils n'ont pas initiée. Pour améliorer la comparabilité des différents débats empiriques dont est tissée la conflictualité sociale sans écraser la singularité de chacun d'eux, il n'est pas seulement utile de distinguer différentes formes agonistiques connexes à la controverse, mais aussi différentes formes de controverses selon leurs contextes d'émergence, leurs enjeux empiriques, leurs acteurs et leurs publics. 


\section{2. \\ Des confrontations politiques agonistiques institutionnalisées?}

Les questions clivantes énumérées en ouverture de cet article portent sur l'instauration, la modification ou la suppression d'une mesure concernant une collectivité, que cette mesure soit relative à l'engendrement, la santé publique, la répartition des richesses, la sécurité, l'alliance ou les droits civiques. Je propose de qualifier de "politiques " ces débats qui voient se confronter publiquement des jugements faisant valoir qu'une situation ou une proposition donnée est contraire à un principe de justice et/ou d'intérêt général, et des jugements inverses sur la même situation ou proposition ; l'enjeu de cette confrontation discursive étant le maintien ou la suppression de la situation ou du projet controversé. Dit autrement, la dimension politique d'une controverse n'est pas liée à certains objets de débat qui seraient par nature politiques; elle est l'aboutissement d'un processus par lequel des acteurs problématisent et publicisent une situation ou un projet quelconque comme ayant des enjeux d'(in)justice ou d'intérêt général. Cette publicisation peut contribuer à son tour à la politisation de nouveaux espaces et de nouveaux acteurs : lorsqu'une question clivante acquiert une publicité croissante via sa réitération dans une diversité d'arènes, l'injonction à avoir une opinion sur la question clivante tend elle-même à s'imposer de façon croissante dans les situations de communication les plus diverses, qu'il s'agisse de conversations au bistrot, d'un repas de famille ou d'un entretien dans les médias ${ }^{6}$.

Depuis les travaux de Jürgen Habermas (1978 [1962]) et les recherches historiques menées dans son sillage, on s'accorde à mettre en relation le développement de l'expression publique d'opinions dissensuelles sur des questions politiques, esthétiques et philosophiques, dans les sociétés allemande, française et anglaise du XVIII ${ }^{\mathrm{e}}$ siècle, avec l'organisation sociale croissante d'une bourgeoisie lettrée se côtoyant au sein d'espaces de sociabilité marqués par la circulation de l'imprimé : cafés, sociétés savantes, salons, clubs ${ }^{7}$. Cependant, c'est au cours du dernier tiers du XIXe siècle que se développe, dans les sociétés industrielles occidentales, un nouveau type de controverses politiques bénéficiant d'un public potentiel composé de l'ensemble des membres de la nation et s'inscrivant dans des formes organisées d'expression collective des dissensus.

6 Sur le rôle des questions clivantes dans les processus de politisation au sein des interactions ordinaires, voir Duchesne et Haegel (2003).

7 Parmi les recherches historiques s'appuyant sur le travail d'Habermas tout en en discutant certains points, se référer notamment à Darnton (1991) et Chartier (1991) pour le XVIII e siècle français ; sur les frontières de la sphère publique bourgeoise et les modalités de la parole populaire contestataire à la même époque, voir entre autres Farge (1992). 
Cette institutionnalisation de certaines formes de controverses politiques et l'élargissement de leurs publics tiennent notamment au développement de la scolarisation primaire et de la presse de masse. Ce double processus ne signifie pas seulement l'accroissement considérable du public qui lit ; il favorise également la constitution, au sein des populations stato-nationales, d'une culture politique commune, voire d'une définition partagée des problèmes publics et des questions clivantes à l'échelle nationale ${ }^{8}$. Ces dernières, formulées bien souvent dans la presse de façon binaire (on ne peut qu'être pour ou contre telle ou telle mesure) participent d'une mise en spectacle du politique sous la forme d'un duel entre positions antagonistes ${ }^{9}$. Censée rendre plus captivants les enjeux sociopolitiques pour un lectorat socialement hétérogène, cette mise en spectacle du dissensus qui se développe au fil du XXe siècle tient aussi à la professionnalisation du journalisme et à l'idéologie qui l'accompagne : donner à lire et à voir des positions opposées en les symétrisant participe d'une mise en scène de la neutralité et de l'objectivité journalistiques, érigées en critère normatif du bon exercice de la profession.

On ne saurait pourtant réduire les controverses politiques à une invention médiatique liée au développement de la presse commerciale de masse et à l'alphabétisation des populations : qu'elles portent sur le droit des femmes, l'instruction publique, la construction de la tour Eiffel, la maltraitance des animaux domestiques ou l'interdiction de la corrida, les controverses politiques qui occupent la presse française des années 1880 sont en relation avec des organisations et des mobilisations collectives. Elles bénéficient de l'institutionnalisation des formes d'expression publique directe et indirecte des opinions, qu'il s'agisse de la législation garantissant la liberté de la presse, du droit de grève et de manifester ou, pour les hommes français majeurs, du droit de vote. Même si ces outils d'expression directe et indirecte des opinions, fruits de luttes sociales et de compromis politiques, sont eux-mêmes l'objet de restrictions et de contre-offensives qui en fragilisent l'usage, ils contribuent à la mise en forme et à la publicisation des clivages et des dissensus qui traversent la population.

L'institutionnalisation de l'expression publique des antagonismes, précisément parce qu'elle est organisée, contrôlée et régulée, ne produit pas, dans les sociétés garantissant ces droits, une prolifération tous azimuts du débat public : certaines normes et valeurs sacralisées, certaines décisions gouvernementales prises dans des contextes de crise, de guerre ou d'« état d'urgence ", sont soustraites à la discussion publique; certaines corporations professionnelles et certaines institutions, par exemple celles qui représentent la " neutralité de l'État », sont tenues de rester à l'écart de la politisation ; enfin et

8 Sur les conditions matérielles de la constitution de cultures politiques nationales à partir du dernier tiers du XIXe siècle, voir par exemple Charle (2011).

9 Sur la mise en forme des débats publics dans la presse française des années 1880, voir par exemple Angenot (1989, chap. 31). 
surtout, certains groupes sociaux ne parviennent pas, par manque de légitimité ou de ressources, à faire entendre leur voix. Dit autrement, du point de vue d'une sociologie de la participation, il ne suffit pas d'énoncer que l'existence d'échanges agonistiques publics est une marque du caractère démocratique d'une société (Amossy, 2014), il convient d'enquêter concrètement sur la nature et le périmètre des questions qui accèdent au rang d'objet du débat public, sur les arènes concernées par ce processus de politisation et sur le statut de celles et ceux qui y participent. Dans une perspective de théorie politique dont peut se nourrir l'analyse sociologique et discursive des controverses politiques, c'est une idée analogue qu'exprime la philosophe Chantal Mouffe (1994) lorsqu'elle met en relation la démocratisation d'une société avec l'élargissement des enjeux, des types d'acteurs et d'actrices ainsi que des arènes de confrontations agonistiques.

\section{3. \\ Controverse politique, controverse scientifique}

Dans les controverses publiques portant sur des questions politiques clivantes, ce sont, comme on l'a vu, principalement les valeurs du juste et de l'intérêt commun - et leurs revers dénonciateurs : l'injuste, l'inutile et le nuisible - qui sont mobilisées par chacun des partis qui s'opposent. Du point de vue de leur axiologie, on pourrait ainsi distinguer ce type de controverses de celles dans lesquelles les protagonistes prennent plutôt appui sur des valeurs esthétiques (se disputant par exemple sur la qualification d'une création artistique comme chef-d'œuvre ou comme imposture) ou sur des valeurs de vérité (se disputant à propos de l'existence ou de l'interprétation d'un phénomène donné). Dans la pratique, ces axiologies peuvent être successivement mobilisées dans une même controverse. Les valeurs invoquées pour s'opposer à la construction d'une œuvre monumentale dans un espace urbain peuvent être successivement politiques et esthétiques. Quant aux controverses environnementales ou bioéthiques, les désaccords qui les caractérisent peuvent porter simultanément sur des enjeux politiques, scientifiques et/ou techniques. Cette hybridité est devenue elle-même l'objet d'un important courant de recherche à I'intersection de la sociologie des sciences et des mobilisations (Pestre, 2007, 38-42, Barthe et Borraz, 2011).

Même au sein de controverses politiques dont les enjeux semblent sans rapport avec les résultats d'enquête scientifique, il se produit fréquemment des dissensus concernant la vérité ou la fausseté de savoirs mobilisés par les débatteurs à l'appui de leurs prises de position : au tournant des XIX $\mathrm{X}^{\mathrm{e}} \mathrm{X} \mathrm{X}^{\mathrm{e}}$ siècles, les études sur les différences entre cerveaux masculins et féminins deviennent enjeux de débat entre féministes et antiféministes s'affrontant à propos de I'accès des femmes au barreau (Rennes, 2007a, 165-190). Un siècle plus tard, 
des recherches en sciences sociales et des théories psychanalytiques sur le genre, la sexualité et la famille, sont invoquées dans les controverses portant sur l'union et la filiation des couples de même sexe (Stambolis-Ruhstorfer, 2015). Ainsi, les désaccords proprement scientifiques, c'est-à-dire portant sur des savoirs non stabilisés (Latour, 1989) et les désaccords proprement politiques, c'est-à-dire mettant en jeu la définition de normes sociales, peuvent correspondre à différentes phases ou dimensions d'une même controverse.

On peut également distinguer les controverses selon les arènes où elles se déploient et le type d'acteurs et d'actrices qui s'y engagent. De ce point de vue, selon la définition proposée par Dominique Raynaud, qui se fait l'écho de maintes recherches en sociologie des sciences, une controverse scientifique est une " division persistante et publique de plusieurs membres d'une communauté scientifique, coalisées ou non, qui soutiennent des arguments contradictoires dans l'interprétation d'un phénomène donné » (Raynaud, 2003, 8). Dans un article qui fait désormais référence, Cyril Lemieux propose de la controverse en général une définition qui n'est pas très éloignée de la définition d'une controverse scientifique :

× À l'intérieur de la « famille » des conflits triadiques [i.e. composé des deux partis du conflit et du public], les controverses semblent se distinguer nettement par la composition très sélective du public qu'elles mobilisent [...]. Mais qu'en revanche, le public sollicité s'élargisse au «plus grand nombre " - autrement dit, aux profanes et aux non-spécialistes - et la controverse [tendra] à se muer en ce que nous serons plutôt tentés de voir comme une " crise institutionnelle » (Lemieux, 2007, 196).

Une controverse est, en somme, " un conflit triadique dans lequel le seul juge est le public des pairs ", ce qui implique, précise Lemieux, des espaces sociaux autonomes où des agents développent « des compétences distinctives au jugement qui leur permettront de se reconnaître mutuellement comme formant un public de pairs par opposition à la masse des profanes » (Lemieux, 2007, 196).

À condition de garder à l'esprit qu'une telle distinction entre pairs et profanes est elle-même susceptible d'être renégociée et de constituer à son tour un enjeu de dispute (Callon, 1981), cette définition de la controverse, qui met en son centre le statut des acteurs qui y participent, est heuristique pour décrire le déroulement de débats qui demeurent confinés ou les phases de confinement de certains débats. Nombre de controverses scientifiques, religieuses, esthétiques et littéraires peuvent en effet être décrites à partir d'une telle définition dès lors que leurs enjeux cognitifs ou épistémologiques ne sont ni appropriés ni même connus au-delà de la communauté de spécialistes au sein de laquelle elles ont émergé, ni retraduits en termes de justice, d'intérêt général ou d'action publique.

Cependant, ces controverses confinées ne peuvent servir que partiellement 
de modèle lorsque l'on constitue des corpus pour enquêter sur les échanges agonistiques portant sur des questions politiques clivantes. En effet, d'une part, les controverses politiques ne se déroulent pas principalement au sein d'une sphère autonomisée de production de savoir, ni même d'une sphère autonomisée de production de la décision publique; d'autre part, leurs énonciateurs ne se définissent pas exclusivement à partir du distinguo entre spécialistes et profanes. Certes, dans les controverses politiques, celles et ceux que Becker appelle les « entrepreneurs de morale » (1985 [1963], 171 sq.), peuvent s'ériger en spécialistes de normes sociales à promouvoir ou à défendre. Tout comme les savants engagés dans des débats politiques clivants, ces entrepreneurs de morale peuvent chercher à se distinguer des profanes par une forme d'expertise, même si celle-ci ne porte pas sur des faits scientifiquement connaissables et validables par des pairs, mais sur la normativité sociale. Cependant, même en prenant en considération ces entrepreneurs de morale, l'opposition expert/profane est loin d'organiser dans son ensemble le droit d'entrée dans les controverses politiques. Des individus peuvent y intervenir comme "victimes " ou « bénéficiaires " potentiels du projet ou de la situation en débat ou encore comme simples citoyens concernés par les questions de justice ou d'intérêt commun que soulève la controverse. Or ce type de configuration - un débat sur une question politique clivante engageant une pluralité d'arènes et de protagonistes ne se catégorisant pas exclusivement par rapport à l'opposition expert/profane - tend bel et bien à être décrit, dans de nombreux travaux de sciences sociales, comme une "controverse " dès lors que les désaccords sont argumentés, publicisés, polarisés et durables ${ }^{10}$.

\section{4. Politisation et dispersion : constituer des matériaux d'enquête}

Si l'on admet que toute politisation s'accompagne d'une dispersion des arènes et des acteurs prenant position sur la question clivante, étudier une controverse politique implique de se distancier d'un conseil couramment donné aux étudiant.e.s voulant constituer un corpus de positions antagonistes sur une question politique : pour comparer des prises de position, il vaudrait mieux que celles-ci soient représentées par des sous-corpus de même taille et inscrits

10 Parmi les travaux sur des questions politiques clivantes qui mobilisent le terme de controverse comme catégorie d'analyse, voir par exemple Phillips (1999), Terzi et Bovet (2005), Laborde (2008), Smadja (2012) Julliard et Cervulle (2013) et Charaudeau (2015) ainsi que les nombreux enseignements sur l'analyse des controverses dispensés dans les études de sociologie, de science politique, les écoles de journalismes et les formations en sciences de l'information et de la communication. Sur ce point, se référer, par exemple, à Badouard (2015). 
dans une même situation de communication ou un même genre discursif, tel un forum de discussion, une assemblée délibérative ou encore des quotidiens nationaux. Cette précaution méthodologique est fondée non seulement pour pouvoir comparer les régularités lexicales ou syntaxiques des différents sous-corpus (par exemple à travers des approches textométriques), mais aussi pour interpréter les différences entre ces sous-corpus. Dès lors que ces derniers appartiennent à un même cadre générique et situationnel, les frontières qui organisent le dicible et l'indicible, les règles tacites ou explicites qui encadrent les formes de l'expression et donc la formulation des désaccords, tendent à être partagées par les énonciateurs. Ainsi, on risque moins de confondre ce qui relève de différences de positionnement politique entre les protagonistes du conflit et ce qui tient au fait que, dans des situations de communication distinctes, les énonciateurs expriment leurs points de vue différemment. Cependant, s'en tenir à cette consigne, en restreignant la prolifération des prises de position à telle ou telle arène du débat public ou à tel ou tel genre discursif, et en corrigeant, par la mise en équivalence quantitative des positions " pro " et "contra ", leur asymétrie dans l'espace social, ne permet pas d'enquêter sur le processus de politisation d'une controverse.

En effet, au fil de ce processus, non seulement les différentes positions s'expriment potentiellement dans l'ensemble des sphères de l'activité sociale, mais en outre, les partisans et les adversaires de la mesure controversée s'expriment rarement dans les mêmes situations d'énonciation ni à parts égales. Ainsi, les controversistes qui soutiennent les revendications politiques les plus hétérodoxes ont généralement moins de probabilité que ceux dont les prises de position coïncident avec la définition hégémonique de la réalité sociale de voir leurs positions relayées au gouvernement, au Parlement, dans les discours d'information des médias dominants ou dans les publications des grands éditeurs. Les " hétérodoxes » auront alors davantage recours à des supports d'édition et de diffusion de leurs idées créés par eux-mêmes et à des modalités d'énonciation collective et/ou anonyme (tract, slogan, pétition, lettre ouverte, inscription murale...). De fait, bien antérieurement à l'usage de l'internet comme outil de communication militante, l'histoire des confrontations sur des questions politiquement clivantes est inséparable de la création, en marge des médias dominants, de supports d'expression et de diffusion des idées contestataires, que ce soit sous une forme verbale, iconique ou audiovisuelle, orale ou écrite ${ }^{11}$.

Ainsi, au fil de la controverse sur l'accès des femmes aux professions traditionnellement masculines qui se développe entre les années 1870 et 1930 en France, les féministes, pour exprimer publiquement leurs positions, créent des associations, des organes de presse défendant le droit des femmes et éditent des brochures spécifiquement dédiées à leur cause. Une grande partie

11 Sur l'histoire du « médiactivisme » depuis les années 1960, voir Cardon et Granjon (2014 [2010]). 
de leurs adversaires, que l'on commence, à la fin du XIX $X^{e}$ siècle, à appeler les " antiféministes " ou les " masculinistes », interviennent au contraire dans les grands quotidiens, publient des essais chez les principaux éditeurs parisiens et ont des alliés qui défendent leur cause dans les lieux du pouvoir politique et académique sans avoir besoin de se constituer des organes spécifiques d'expression des idées antiféministes (Rennes, 2007a, 9-15). C'est seulement dans une société où le " masculinisme » n'est plus dicible dans les médias dominants et les instances de pouvoir, qu'il est susceptible de se constituer en cause spécifique avec ses associations, ses journaux, ses maisons d'édition. En revanche, on chercherait en vain, pour constituer un corpus permettant de rendre compte de la controverse des années 1870-1930 sur les droits des femmes, une symétrie des genres discursifs mobilisés par les deux partis (par exemple deux journaux militants antagonistes occupant une position homologue dans le champ journalistique) ou un espace commun où ces deux partis pourraient s'exprimer de façon équivalente (par exemple un grand quotidien national).

Dans une enquête sur une controverse politique, le suivi minutieux des mentions du discours adverse au sein de chaque position dessine au contraire les contours d'un espace énonciatif agonistique fondamentalement asymétrique et hétérogène (du point de vue de la situation d'énonciation et de la " taille » des différents protagonistes), et non synchronisé (du point de vue de la temporalité des affrontements). Ainsi, dans la controverse que je viens d'évoquer, des parlementaires s'en prennent, en séance publique, à une série d'articles parue plusieurs semaines auparavant dans un petit journal féministe, tandis que des militantes du droit des femmes organisent une action collective devant un théâtre où se joue une comédie dont le principal argument narratif exprime une position hostile à l'accès des femmes aux professions traditionnellement masculines (Rennes, 2007a, 107-127). Parmi les adversaires des revendications féministes, les formes d'intervention agonistiques sont elles-mêmes très hétérogènes et sont grandement dépendantes des ressources statutaires et socio-économiques des acteurs mobilisés. Ainsi, les médecins et les avocats produisent des argumentaires dans la grande presse, et par l'intermédiaire de leurs alliés, au Parlement ou au conseil municipal, pour soutenir ou, le plus souvent, pour dénoncer les demandes d'ouverture aux femmes de leurs corporations respectives. Ils parviennent ainsi à transformer en question de justice et d'intérêt général leurs désaccords internes sur la mixité du barreau et de la médecine. En revanche, d'autres professionnels comme les cochers, eux aussi en majorité hostiles à ce que des femmes exercent leur métier, ne disposent pas des mêmes ressources pour transformer leurs problèmes corporatifs - l'arrivée des premières cochères - en controverse publique : il faut alors consulter la presse syndicale des cochers ainsi que les archives policières et judiciaires où sont répertoriés des accidents malveillants, que certains provoquent contre les nouvelles conductrices, pour saisir des traces de protestations contre l'arrivée des femmes (Rennes, 2016). 
Comme on le voit à travers ces exemples, qui nous confrontent à la frontière entre controverse publique et conflit social, prendre en considération la dispersion, l'hétérogénéité et les formes d'asymétries des interventions agonistiques nous amène à explorer la dimension multimodale de ces dernières et donc à interroger les articulations possibles entre analyse des controverses publiques et sociologie des conflits sociaux et de l'action collective. Nombre d'actions collectives obtiennent gain de cause, échouent ou s'étiolent sans qu'aucune controverse publique ne se développe en rapport avec l'objet de la revendication : en ce sens, bien des enquêtes sur des mobilisations peuvent tout à fait se passer des outils de l'analyse des controverses. La réciproque n'est pas vraie : une controverse sur une question politique clivante peut difficilement perdurer sans acteurs coalisés et sans un continuum d'interventions agonistiques qui ne sont pas réductibles à la seule production d'argumentaires verbaux. Les controverses politiques portant sur les droits des minorités sexuelles et sur l'enseignement de la " théorie du genre », qui ont marqué plusieurs pays européens au tournant des $X X^{e}-X_{X I}$ siècles, s'inscrivent par exemple toutes dans le cadre plus large de mobilisations et de contre-mobilisations incluant diverses techniques de protestation collectivement organisées (occupation, manifestation, mobilisation " rhétorique » de pratiques corporelles... ${ }^{12}$ ) qui sont elles-mêmes conçues par leurs initiateurs comme ayant une dimension argumentative dans le conflit (Rennes, 2011a, 152-161).

Cependant, étudier le processus par lequel des éléments de discours adverses sont repris, reformulés et contestés de façon multimodale par des acteurs hétérogènes, comporte le risque de se confronter, au fil de l'enquête, à des corpus potentiellement illimités. Dit autrement, on ne saurait adopter l'interdiscursivité - de surcroît envisagée dans une perspective multimodale - comme seul critère de sélection des matériaux d'enquête. D'autres critères de délimitation sont, de fait, compatibles avec l'étude de la dispersion et de la politisation : suivre dans une diversité d'arènes, la circulation interdiscursive d'une formule ou d'un type d'argument particulier ${ }^{13}$ ou encore se concentrer sur l'interdiscursivité qui se déploie au sein d'un épisode ou d'un évènement circonscrit de la controverse, permet par exemple d'éviter de se confronter à une masse incontrôlable de matériaux sans pour autant délimiter a priori les corpus en terme de genre discursif ou de situation de communication.

En outre, une enquête sur la dispersion n'implique pas de renoncer à la perspective d'inclure également dans l'enquête des sous-corpus délimités selon des critères génériques ou communicationnels. L'étude exhaustive d'un sous-corpus, composé par exemple de l'ensemble des tribunes d'un grand

12 L’usage du corps - nu, attaché, bâillonné, déguisé ou masqué... - comme métaphore d'un abus de pouvoir ou d'une injustice est beaucoup étudié dans le courant de la « rhétorique des mouvements sociaux ». Voir par exemple DeLuca (1999).

13 Sur ce type de recherche voir Ebel et Fiala (1983) et Krieg-Planque (2009). 
quotidien national ou de l'ensemble des débats parlementaires sur la question clivante, permet d'objectiver non seulement les asymétries entre les différentes positions au sein d'institutions discursives stratégiques mais aussi, si le corpus est constitué en sous-parties chronologiques, l'évolution de ce rapport de forces. Cependant, un tel corpus ne saurait être considéré comme suffisant pour analyser le processus de politisation et donc de dispersion qui caractérise les controverses politiques.

\section{5. Affaire, problème public}

Telle que présentée jusqu'ici, la politisation d'une question clivante comporte un certain nombre de caractéristiques communes avec le processus de développement d'une " affaire », analysé en particulier par Elizabeth Claverie $(1994,1998)$. Telle qu'elle la conçoit, la notion d'affaire doit être pensée en relation à la fois avec le commérage et le scandale. Si un individu ou un collectif transgresse des valeurs qu'il est censé honorer - que ce soit dans le cadre d'activités politiques, professionnelles, financières, familiales... - et si cette transgression est connue par un nombre limité de personnes, elle peut se limiter à susciter de simples " commérages " au sein d'un réseau d'interconnaissances. Si la révélation d'une telle transgression s'élargit au-delà de ce réseau et conduit à une désapprobation publique de la conduite transgressive, elle devient un « scandale » (Dampierre, 1954 ; Thompson, 2000 ; Blic et Lemieux, 2005).

Par la suite, si certaines personnes se désolidarisent publiquement de cette désapprobation, défendant l'individu ou le collectif accusé contre ses accusateurs au point qu'un clivage public se produit quant à l'innocence ou la culpabilité de l'accusé, nous sommes face à une " affaire ". Ainsi, en France, dans I'affaire Dreyfus en 1894, Russier en 1969 ou Strauss-Kahn en 2011, un clivage durable se cristallise sur la qualification des actes incriminés et sur l'attribution des places de coupable ou de victime ${ }^{14}$. Comme dans la constitution d'une controverse politique, la confrontation discursive qui se noue autour de l'affaire est publique, argumentée et polarisée, et peut être source de politisation des interactions ordinaires.

Cependant, une affaire ne se transforme en controverse politique qu'à la double condition que se constitue d'abord une revendication politique, détachée de la personne singulière autour de laquelle s'est cristallisée l'affaire, puis que cette revendication suscite la formation durable de deux camps antagonistes : par exemple, une affaire de mœurs débouche sur une revendication concernant

14 Cette distinction entre "scandale » et " affaire », qui constitue une proposition opératoire pour l'analyse de la conflictualité publique, n'est pas une description des usages ordinaires de ces deux termes, souvent employés comme des synonymes. 
la législation en matière sexuelle qui devient à son tour une question clivante du débat public. Ce processus de transformation d'une affaire en controverse politique n'est pas exceptionnel : en France, le débat sur le " port du voile » dans les écoles publiques se constitue initialement à partir d'une affaire incarnée par trois jeunes filles et par un responsable d'établissement scolaire en 1989, avant de devenir, pendant plus de vingt ans, une question clivante problématisée comme un enjeu d'action publique autonomisé par rapport aux cas initiaux qui ont accompagné son émergence.

Cent ans auparavant, un processus en partie analogue se produisit à propos de l'accès des femmes au métier d'avocat : d'abord incarnée par un cas précis - "l'affaire Jeanne Chauvin » du nom de la première diplômée en droit ayant postulé au barreau de Paris en 1897 -, la question de la mixité du barreau devient, entre 1898 et 1900, un débat national clivant se déployant à la fois au sein du Conseil de l'ordre des avocats, dans tous les grands quotidiens, dans des essais, sur des scènes de théâtre de boulevard, dans des fictions, des chansons, l'ensemble de la culture visuelle jusqu'à la Chambre des députés et au Sénat où est finalement votée une loi autorisant les femmes à devenir avocates (Rennes, 2007a).

Ainsi, les formes « affaire » et " controverse " peuvent correspondre à différents moments dans la carrière d'une question clivante ou différentes dimensions de cette question. Mais toutes les affaires ne se transforment pas en controverses, pas plus que toutes les controverses politiques ne se déploient à partir d'affaires préalables : une proposition législative, une action collective, la prise de position d'une personnalité publique ayant un accès routinisé aux grands médias, la publicisation d'une situation problématique par un « lanceur d'alerte " sans notoriété (Chateauraynaud et Torny, 1999) peuvent contribuer à l'émergence d'une controverse sans qu'il n'y ait eu préalablement d'affaire localisée et incarnée.

Le fait qu'une controverse politique, à la différence d'une affaire, porte directement sur un enjeu d'action publique semble la rapprocher du « problème public " tel qu'analysé en sociologie politique. La constitution d'un problème public est, elle aussi, jalonnée de dissensus : ceux-ci sont présents dès le processus de définition d'une situation comme étant " anormale " et comme nécessitant une intervention d'un acteur public (Muller et Surel, 2000, 57). Par exemple, l'identification des causes et des conséquences du problème, la catégorisation de ses responsables et de ses victimes, la façon d'y mettre fin et l'identification des acteurs légitimes pour le faire sont l'occasion de cadrages et de contre-cadrages (Benford et Hunt, 2001 [2000] ; Gusfield, 2012 [1989]). Cependant, dans une controverse politique, le clivage se situe en amont de la mise en intrigue d'une situation définie comme problématique : il porte sur le statut même de problème de ladite situation, au sens où celle-ci est cadrée comme un problème par l'un des partis de la controverse et comme un fait juste et nécessaire par le parti adverse. Ainsi, dans les « controverses d'égali- 
té » (Rennes, 2007b, 2007c, 2011b), le fait qu'une catégorie de population (les femmes, les mineurs, les étrangers, les homosexuels...) n'ait pas légalement accès à une ressource donnée constitue pour les uns un problème de justice, alors que pour les autres, une telle restriction est justifiée par l'intérêt général et des différences essentielles entre les groupes juridiquement distingués.

Au fil de la carrière d'une controverse et de l'évolution du rapport de forces entre les protagonistes, il peut arriver que la position consistant à définir la situation comme problématique devienne hégémonique tandis que la définition adverse devienne illégitime : la controverse s'est alors transformée en problème public. Ainsi, à partir des années 1980, la pédophilie est de plus en plus problématisée comme une situation anormale nécessitant des mesures de la part des pouvoirs publics. Des débats se développent quant aux causes de cette pratique, quant au nombre de ses victimes, quant à la hiérarchie des mesures nécessaires pour l'endiguer. Pour autant, cette question devenue problème public n'oppose pas des partisans et des opposants de la pédophilie, comme peuvent s'opposer, dans les années 2000-2010, des défenseurs et des adversaires du mariage des couples de même sexe, de la liberté de porter un voile à l'école ou de la légalisation du cannabis. En effet, le droit des adultes à avoir des relations sexuelles avec des enfants, dicible et audible dans la France des années 1970 (Berard et Sallé, 2015), est devenu indéfendable dans le débat public à la fin des années 1980 (Boussaguet, 2008). Ce qui aurait pu constituer une controverse durable sur l'autorisation de relations sexuelles entre adultes et enfants s'est converti en débat sur les moyens de lutter contre ce qui est défini, de façon dominante, comme le problème de la pédophilie.

6.

\section{Les frontières du publiquement dicible}

L'exemple de la constitution de la pédophilie comme problème public nous renvoie à un dernier point qu'il convient d'expliciter : une controverse politique implique le caractère publiquement dicible d'au moins deux points de vue opposés. Dit autrement, toute controverse politique se déploie dans le cadre d'un espace doxique définissant une série limitée de questions débattables dont la formulation même charrie un certain nombre de présupposés qu'il faut accepter pour s'engager dans le débat.

Ainsi en va-t-il des « controverses d'égalité » que j'ai évoquées plus haut: celles-ci portent sur le fait de décider s'il est justifiable que l'accès légal à une institution (le droit de se marier, de voter, d'exercer telle profession, de concourir à une compétition sportive, de recevoir une récompense honorifique, d'utiliser tel transport public, etc.), soit réservé à une catégorie donnée de la population (les nationaux, les hommes, les titulaires de tel diplôme, les femmes de tel âge, les couples hétérosexuels, les personnes de telle taille, telle religion, telle couleur de peau...) ou s'il doit concerner une catégorie plus 
large. Participer à ce type de controverse implique de s'accorder sur le fait que l'institution, dont le périmètre d'accès est en débat, constitue elle-même un bien. En effet, celles et ceux qui la dénoncent comme partie-prenante d'un système dont on doit souhaiter l'abolition ne peuvent participer à la controverse dans les termes où elle se pose : si l'on milite pour l'abolition du mariage, de la Légion d'honneur, des " grandes écoles » ou des transports polluants, il est difficile de s'engager dans un débat portant sur l'extension des bénéficiaires de l'accès à ces ressources.

Face aux controversistes qui s'accordent sur les termes du débat, ceux et celles qui les refusent sont dans la position de ce que Jean-François Lyotard nomme un " différend " (Lyotard, 1983, 25) : leur distance sociale, politique et/ ou cognitive est telle avec les protagonistes du débat que leurs oppositions ne peuvent s'énoncer dans un « idiome commun ». Lorsque l'instauration ou la suppression d'une mesure concernant une collectivité est en jeu, les positions tierces, qui tendent à mettre en cause l'alternative en débat, se voient souvent marginalisées au profit de la constitution de deux camps antagonistes : les " pour » et les « contre ». La polarisation prend ainsi souvent la forme d'une bipolarisation, laquelle s'accompagne également de stratégies rhétoriques de " dichotomisation », par lesquelles chacun des camps souligne "l'incompatibilité des deux pôles ", "le bien-fondé de la réponse qu'il prône " et "l'inexistence de toute alternative » traduction de l'auteure (Dascal, 2008, 34).

Pour comprendre ce périmètre du publiquement débattable, il ne suffit pas d'analyser ce qui est commun, en termes de système de valeurs et d'éléments doxiques, aux adversaires qui s'affrontent dans une controverse (Rennes, op. cit., 2007a, 369 sq.). Il convient également de s'intéresser aux acteurs, aux objets et aux prises de position qui sont hors de la controverse publique. Dans L'Ordre du discours, Michel Foucault (1971, 11-12) identifie trois systèmes d'interdit qui organisent la production du discours et en limitent la prolifération : les sujets « tabous » (" on sait bien que l'on n'a pas le droit de tout dire »), les «circonstances " (" on ne peut pas parler de tout dans n'importe quelle circonstance ») et le statut du sujet qui parle (" n'importe qui, enfin, ne peut pas parler de n'importe quoi »). Cette hypothèse très générale sur les règles de limitation du dicible peut constituer un point de départ pour enquêter sur les formes de conflictualité discursive autorisées dans une société donnée.

Une telle question de recherche implique de ne pas constituer des corpus uniquement composés de discours publiquement disponibles car déjà publicisés par des militants, des journalistes, des éditeurs, des professionnels de la politique. Les essais, les pamphlets, les tribunes dans la presse, les discours gouvernementaux et parlementaires, les tracts, les pétitions, les manifestes et, depuis les années 2000, les sites web sont généralement les premiers corpus vers lesquels on se tourne quand on s'intéresse à une controverse politique. Or, les "contre-discours ", produits par celles et ceux que Nancy Fraser appelle les « contre-publics » qui ne peuvent exprimer leurs idées et 
mettre en forme leurs expériences " au sein des publics dominants » (Fraser, 2001 [1992], 138-139) doivent être traqués dans des matériaux d'enquête n'ayant pas un tel degré de publicité : archives associatives, policières, judiciaires ou privées, entretiens réalisés avec des activistes dans le cadre de la recherche, observation ethnographique d'espaces d'entre-soi militants où s'échangent des propos métadiscursifs sur ce qui peut être dit, et ce qui doit être tu, en dehors de ces espaces et où s'élaborent des revendications avant qu'elles ne se frayent un chemin vers des publics plus diversifiés ${ }^{15}$. Bref, il s'agit de constituer des matériaux d'enquête, en deçà et à côté des discours publicisés, pour appréhender les prises de position qui sont demeurées durant un temps donné, ou demeurent encore pour une période indéterminée, dans les coulisses de la contestation publique.

\section{7. Conclusion}

À partir d'une exploration des travaux sur les échanges agonistiques et de mes propres recherches, j'ai proposé de distinguer les controverses de formes parentes, notamment le débat et la polémique, puis de préciser en quoi consistent les frontières et les recouvrements entre controverses scientifiques et politiques, et enfin entre les controverses politiques et ces autres formes de conflictualité politique que sont "l'affaire " et le "problème public ". En définissant une controverse politique comme une confrontation argumentée, polarisée, dispersée dans plusieurs sphères de l'activité sociale et portant sur un enjeu d'action publique, l'objectif n'était pas de substantialiser la controverse politique, mais de rendre comparables différentes formes agonistiques et de pouvoir analyser la réitération, la pérennisation et la politisation de certains débats : si l'on appelle « politisation » le processus par lequel une diversité d'acteurs, dans une diversité de sphères sociales, prend position sur une question clivante au nom d'un principe de justice ou d'intérêt général, on risque de rater la compréhension de ce processus en se restreignant d'emblée à l'analyse des arènes du débat les plus directement liées à la décision publique, à l'expertise et aux grands médias.

Quels que soient les domaines de l'action publique dans lesquels s'inscrivent les controverses, analyser ces dernières permet plusieurs types de

15 Sur l'ethnographie des espaces militants, voir notamment Cefaï et al. (2012). Quant au cheminement des revendications depuis des espaces d'entre-soi militants vers des publics élargis, il constitue l'objet de l'enquête collectivement menée au sein du séminaire "Argumenter, revendiquer, contester » que j'anime à l'EHESS depuis 2014 : Rennes J. (2015-2016). «Argumenter, revendiquer, contester : analyses de discours, théories de l'argumentation et sociologie des mobilisations ». In EHESS: Base de données des enseignements et séminaires de l'EHESS, [En ligne], <http://enseignements-2015.ehess.fr/2015/ ue/326/>. 
contribution à la sociologie politique et aux disciplines du discours, par-delà chaque cas étudié. Tout d'abord, l'analyse des controverses politiques permet d'éclairer à la fois les conflits de valeurs qui traversent une société et l'espace doxique commun sur lequel s'élèvent les désaccords et les prises de décisions publiquement énonçables. Deuxièmement, l'analyse des controverses politiques, tout comme celle des affaires et des problèmes publics, peut devenir un mode d'entrée dans les processus de politisation dès lors que les questions clivantes deviennent des objets de conversation et des occasions de positionnement au sein des interactions ordinaires. Enfin, travailler sur des controverses politiques peut constituer une contribution à la mise en relief de l'historicité des politiques publiques, tout comme l'étude des controverses scientifiques nous révèle I'historicité du champ scientifique et des connaissances qui y sont produites. En effet, suivre la carrière d'une controverse scientifique ou politique qui a présidé au développement de connaissances ou de mesures d'action publique désormais légitimes conduit à appréhender, dans la durée, la transformation des rapports de force et des jeux d'alliance qui ont contribué à l'établissement de nouvelles orientations scientifiques ou politiques. Or, la genèse conflictuelle des politiques publiques est souvent occultée, non seulement par le processus ordinaire de naturalisation des institutions, mais aussi parce que ceux qui, dans les instances de pouvoir, se sont opposés à des revendications désormais transformées en lois, en règlements, en droits, ont tout intérêt à faire oublier leurs oppositions tout comme les résistances antérieures de ceux dont ils sont les héritiers politiques.

Il y a ainsi de multiples raisons pour enquêter sur des controverses politiques, à condition de ne pas oublier qu'elles ne sont qu'une partie de la conflictualité sociale : les groupes sociaux ont un pouvoir inégal à transformer les problèmes auxquels ils sont confrontés en controverse publique et certains des clivages qui traversent le monde social ne sont pas dicibles dans les termes du débat public. Il existe, dans les travaux sur les controverses, une tension dans l'extension du terme : tantôt la controverse, envisagée du point de vue de sa carrière, englobe des moments d'éclipse et de renaissance, de visibilité et de reflux de la publicité ; tantôt, la controverse est un moment spécifique dans I'histoire de la confrontation autour d'une question clivante, ce moment où les positions antagonistes sont parvenues à s'énoncer durablement dans une diversité d'arènes publiques. Cependant, pour éclairer les frontières de ce qui est politiquement débattable, peu importe que l'on appelle « controverse " une phase particulière du déploiement d'une question clivante ou l'ensemble de la carrière de celle-ci. Il est, dans les deux cas, heuristique d'explorer non seulement ce qui précède la publicisation de la controverse, mais aussi les marges de celle-ci : les formes de problématisation et de positions qui ne sont plus, ou pas encore, reconnues comme s'inscrivant dans des clivages politiquement légitimes. 
Bibliographie

Amossy R. (2008). « Argumentation et Analyse du discours : perspectives théoriques et découpages disciplinaires ». In Argumentation et Analyse du Discours [En ligne], $\mathrm{n}^{\circ} 1,<$ https://aad.revues.org/200>.

Amossy R. (2014). Apologie de la polémique, Paris, PUF.

Amossy R. et Burger B. (2011). «Introduction : la polémique médiatisée ». In Semen, n³1, p. 7-24.

Angenot M. (1982). La Parole pamphlétaire, Paris, Payot.

Angenot M. (1989). 1889 : un état du discours social, Longueil, Édition du Préambule.

Angenot M. (2012). « La notion d'arsenal argumentatif : I'inventivité rhétorique dans I'histoire ". In Frydman B. et Meyer M. (dirs.), Chaïm Perelman (1912-2012), Paris, PUF, p. 39-68.

Badouard R. (2015). « Enseigner les controverses en école de journalisme ». In Hermès, n 73, p. 51-54.

Barthe Y. et Borraz O. (2011). " Les controverses sociotechniques au prisme du Parlement ". In Quaderni, n 75, p. 63-71.

Becker H. (1985 [1963]). Outsiders, Paris, Métailié.
Benford R. et Hunt S. (2001 [2000]). "Cadrages en conflit ». In Cefai D. et Trom D. (dirs.), Les formes de l'action collective, Paris, coll. "Raisons pratiques ", Éditions de I'EHESS, p. 163-194.

Benoit-Barné C. et McDonald J. (2011). "L'évolution des pratiques rhétoriques de délibération publique par la controverse ". In Communication [En ligne], vol. 28/2, <http:// communication.revues.org/1676>.

Berard J. et Sallé N. (2015). « Les âges du consentement. Militantisme gai et sexualité des mineurs en France et au Québec (19701980) ». In Clio, n 42, p. 99-124.

Blic D. de et Lemieux C. (2005). "Le scandale comme épreuve. Éléments de sociologie pragmatique ». In Politix, n 71, p. 9-38.

Boltanski L. (1984). « La dénonciation ». In Actes de la recherche en sciences sociales, $n^{\circ} 51$, p. 3-40.

Boussaguet L. (2008). La Pédophilie, problème public. France, Belgique, Angleterre, Paris, Dalloz.

Callon M. (1981). " Pour une sociologie des controverses technologiques ». In Fundamenta Scientae, $\mathrm{n}^{\circ}$ 2, p. 381-399.

Cardon D. et Granjon F. (2014 [2010]). Médiactivistes, Paris, Presses de Science Po.

Cardon D., Heurtin J.-Ph. et Lemieux C. (1995). «Parler en public ». In Politix, n 31, p. 5-19. 
Cefai D., Carrel M., Talpin J.,

Eliasoph N. et Lichterman P. (dirs.)

(2012). Dossier «Ethnographies de la participation ». In Participations, $n^{\circ} 4$.

Charaudeau P. (2015). La laïcité dans l'arène médiatique. Cartographie d'une controverse sociale, Paris, INA Éditions.

Charle C. (2011). Discordances des temps, Paris, Armand Colin.

Chartier R. (1991). Les origines culturelles de la Révolution française, Paris, Le Seuil.

Chateauraynaud F. (2011). "Sociologie argumentative et dynamique des controverses : l'exemple de l'argument climatique dans la relance de l'énergie nucléaire en Europe ». In A contrario, $n^{\circ} 16, p .131-150$.

Chateauraynaud F. et Torny D. (1999). Les Sombres Précurseurs. Une sociologie pragmatique de l'alerte et du risque, Paris, Éditions de l'EHESS.

Claverie E. (1994). « Procès, Affaire, Cause : Voltaire et l'innovation critique ». In Politix, n² 26, p. 76-85.

Claverie E. (1998). « La naissance d'une forme politique : I'affaire du Chevalier de la Barre ». In Roussin P. (dir.), Critique et affaires de blasphème à l'époque des Lumières, Paris, Honoré Champion, p. 185-260.

Dampierre E. de (1954). «Thèmes pour l'étude du scandale ". In Annales ESC, IX (3), p. 328-336.

Darnton R. (1991). Édition et sédition :
I'univers de la littérature clandestine au XVIII siècle, Paris, Gallimard.

Dascal M. (2008). « Dichotomies and types of debates ». In Eemeren F. H. van et Garssen B. (dirs.), Controversy and Confrontation. Relating controversy analysis with argumentation theory, Amsterdam, Philadelphia, John Benjamins Publishing Company, p. 27-50.

Dascal M. (1995). « Observation sur la dynamique des controverses ». In Cahiers de linguistique française, $n^{\circ} 17$, p. 99-121.

Desbordes F. (1995). « La place de I'autre. Remarques sur quelques emplois de 'controversia' dans la rhétorique latine ». In Le Boulluec A. (dir.), La controverse religieuse et ses formes, Paris, Cerf, p. 29-46.

DeLuca K. M. (1999). « Unruly Arguments : The Body Rhetoric of Earth First! Act Up and Queer Nation ». In Argumentation and Advocacy, $\mathrm{n}^{\circ}$ 36, p. 9-21.

Duchesne S. et Haegel F. (2003). "Politisation et conflictualisation ". In Perrineau P. (dir.), Le désenchantement démocratique, La Tour d'Aigues, Éditions de l'Aube, p. 107-129.

Ebel M. et Fiala P. (1983). Sous le consensus, la xénophobie. Paroles, arguments, contextes (1961-1981), Lausanne, Institut de science politique.

Eemeren F. H. van et Garssen B. (2008). "Controversy and confrontation in argumentative dis- 
course ». In Eemeren F. H. van et Garssen B. (dirs.), Controversy and Confrontation. Relating controversy analysis with argumentation theory, Amsterdam, Philadelphia, John Benjamins Publishing Company, p. 1-26.

Farge A. (1992). Dire et mal dire au XVIII siècle, Paris, Le Seuil.

Foucault M. (1971). L'ordre du discours, Paris, Gallimard.

Fraser N. (2001 [1992]). « Repenser la sphère publique : une contribution à la critique de la démocratie telle qu'elle existe réellement ". In Hermès, $n^{\circ} 31$, p. 125-156.

Grand Robert de la langue française (2001). "Controverse ", Dictionnaire Le Robert éditions, Paris, p. 554.

Gusfield J. (2012 [1989]). « Significations disputées. Contester la propriété et l'autorité des problèmes sociaux ». In Cefaï D. et Terzi C. (dirs.), L'expérience des problèmes publics, Paris, Éditions de l'EHESS, coll. «Raisons pratiques », p. 113-132.

Gusfield J. (2009 [1981]). La culture des problèmes publics, Paris, Économica.

Habermas J. (1978 [1962]). L'espace public. Archéologie de la publicité comme dimension constitutive de la société bourgeoise, Paris, Payot.

Jacquin J. (2011). « Le/La polémique : une catégorie opératoire pour une analyse discursive et interactionnelle des débats publics ? ». In Semen, n 31, p. 43-60.
Julliard V. et Cervulle M. (2013). "Différence des sexes et controverses médiatiques : du débat sur la parité au mariage pour tous " (1998-2013) ». In Le Temps des médias, $n^{\circ} 21$, p. 161-175.

Kerbrat-Orecchioni C. (1980). "La polémique et ses définitions ». In Le discours polémique, Lyon, PUL, p. 3-40.

Krieg-Planque A. (2009). La notion de "formule " en analyse du discours. Cadre théorique et méthodologique, Besançon, Presses Universitaires de Franche-Comté.

Laborde C. (2008). Critical Republicanism. The Hijab Controversy in Political Philosophy, Oxford, Oxford University Press.

Latour B. (2005 [1989]). La science en action. Introduction à la sociologie des sciences, Paris, la Découverte.

Lemieux C. (2007). «À quoi sert I'analyse des controverses? ». In Mil neuf cent - Revue d'histoire intellectuelle, $\mathrm{n}^{\circ} 25, \mathrm{p} .191-212$.

Lilti A. (2007). «Querelles et controverses. Les formes du désaccord intellectuel à l'époque moderne ". In Mil neuf cent - Revue d'histoire intellectuelle, $\mathrm{n}^{\circ} 25, \mathrm{p} .13-28$.

Lyotard J. F. (1983). Le Différend, Paris, Minuit.

Maingueneau D. (2009 [1996]). Les termes clés de l'analyse de discours, Paris, Seuil, coll. "Point Essai ». 
Mouffe C. (1994). Le politique et ses enjeux. Pour une démocratie plurielle, Paris, MAUSS.

Muller P. et Surel Y. (2000 [1998]). L'Analyse des politiques publiques, Paris, Montchrestien.

Pestre D. (2007). « L'analyse de controverses dans l'étude des sciences depuis trente ans ». In Mil neuf cent - Revue d'histoire intellectuelle, $\mathrm{n}^{\circ} 25$, p. 29-43.

Phillips K. R. (1999). « A Rhetoric of Controversy ". In Western Journal of Communication, $n^{\circ} 63$, p. 488-510.

Plantin C. (2003). « Des polémistes aux polémiqueurs ». In Murat M., Declercq G. et Dangel J. (dirs.), La parole polémique, Paris, Champion, p. 377-408.

Plantin C. (2002). " Argument ». In Charaudeau P. et Maingueneau D. (dirs.), Dictionnaire d'analyse de discours, Paris, Seuil.

Raynaud D. (2003). Sociologie des controverses scientifiques, Paris, PUF.

Rennes J. (2016). « Cochères parisiennes. Le risque en spectacle ». In Travail, Genre et Sociétés, $n^{\circ} 36$, p. 37-59.

Rennes J. (2011a). " Les formes de la contestation. Sociologie des mobilisations et théories de l'argumentation ». In A contrario, n 16, p. 151-173.

Rennes J. (2011b). « Illégitimer des distinctions en droit ». In Politix, n 94, p. 35-57.
Rennes J. (2007a). Le mérite et la nature. Une controverse républicaine, l'accès des femmes aux professions de prestige (1880-1940), Paris, Fayard.

Rennes J. (2007b). « Analyser une controverse ». In Bonnafous S. et Temmar M. (dirs.), Analyse de discours et sciences humaines et sociales, Paris, Ophrys, p. 91-107.

Rennes J. (2007c). " Les controverses d'égalité en droit ». In Badie B. et Déloye Y. (dirs.), Le temps de l'État, Paris, Fayard, p. 408-419.

Scotto d'Apollonia L., Luxardo G. et Piet G. (2014). « Approche lexicométrique des controverses climatiques ». In Communication aux JADT 2014 [En ligne], Paris, <https://hal. archives-ouvertes.fr/hal-01139755>.

Smadja D. (dir.) (2012). Dossier "Penser la controverse ». In Raisons politiques, $\mathrm{n}^{\circ} 47$.

Stambolis-Ruhstorfer M. (2015). La culture du savoir : la construction de "l'expertise » dans les débats politiques sur le mariage et la filiation pour les couples de même sexe en France et aux États-Unis, Thèse de doctorat en sociologie de I'EHESS et de I'UCLA.

Terzi C. et Bovet A. (2005). « La composante narrative des controverses politiques et médiatiques ". In Réseaux, n¹32, p. 111-132.

Thompson J. B. (2000). Political Scandal. Power and Visibility in the Media Age, Cambridge, Polity Press. 


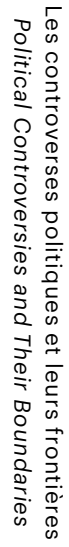


